\title{
Neurologic Side Effects of Psychiatric Treatments
}

\author{
R. J. Dolan, M.D., M.R.C. Psych
}

Little is known in psychiatry concerning funamental disease processes. The relative absence idisease-specific treatments is therefore not surrising. Most physical treatments in psychiatry exert their therapeutic effects in a nonspecific fashion. Lack of disease specificity means that compromises are continually made between beneficial and unwanted treatment effects. Indeed, the goal ff most therapeutic developments, given limitalons in knowledge concerning disease processes, is enhance wanted over unwanted effects.

Physical treatments in psychiatry, as in other ranches of medicine, aim to ameliorate, arrest, and reverse disease processes. In practice, the arrest or reversal of psychiatric disease is an idealistic and rarely achieved goal. In this, psychiatry shares much in common with neurology in that symptom relief is the principal therapeutic aim.

Despite these limitations, there are many benficial and effective treatments in psychiatry. The mediation of these effects is generally ascribed to atterations in neuronal function at neuroreceptor (ites. However, a lack of pharmacologic and disease secificity means that psychiatric interventions are associated with high rates of unwanted effects. Because of their high prevalence, neurologic side effects are the most important hazards of psychiatric treatments. The recognition of neurologic side eflects is therefore of great clinical importance.

This review examines the prevalence, clinical presentation, putative pathophysiology, and mangement of neurologic side effects of psychiatric treatments. It is not possible within the brief space of this article to cover all treatments in psychiatry; therefore the focus will be on the more convenfonal and widespread treatments. These include the neuroleptics, lithium, antidepressants, electroconvulsive therapy (ECT), anxiolytics, and psychourgery.

\section{NEUROLEPTICS}

This broad group of compounds constitutes the cornerstone of pharmacologic treatment of the psychoses, particularly schizophrenia. The introduction of potent antipsychotic agents into clinical practice in 1952 was associated with an almost immediate recognition that these agents could result in neurologic side effects. ${ }^{1,2}$ Side effects may for convenience be thought of as acute and chronic, though in reality such a division is arbitrary and artificial. There is little dispute that neuroleptics cause acute neurologic side effects, but there is controversy as to whether they are either necessary or sufficient causes for the emergence of chronic side effects, in particular tardive dyskinesia (TD). This question will be addressed further in considering TD. The most commonly encountered neurologic side effects of neuroleptics are summarized in Table 1.

\section{ACUTE DYSTONIC REACTIONS}

Acute dystonic reactions were first reported in association with chlorpromazine treatment. Because of their distribution, they were labeled "facio-bucco-linguo-masticatory dyskinesia" and the similarity with "excito-motor" syndromes seen in the wake of epidemic encephalitis was noted. ${ }^{3}$ These reactions may be focal or generalized. Their onset, which is abrupt, is associated with com-

\section{Table 1. Neurologic Side Effects of Neuroleptics}

Acute dystonic reactions

Akathisia

Parkinsonism

Tardive dyskinesia

Tardive dystonia

Neuroleptic malignant syndrome

Consultant Psvchiatrist, National Hospital for Nervous Diseases, Qucen Square, London, and Senior Lecturer, Roval Free School of Medicine, Pond Screet, London, United Kingdom

Reprint requests: Dr. Dolan, National Hospital for Nervous Diseases, Queen Square, London, WCIN $3 B$ B UK

Copyright 1990 by Thieme Medical Publishers, Inc., 381 Park Avenue South, New York, NY 10016. All rights reserved 
mencement or increments of treatment. The manifestations are varied and extremely distressing to affected patients. The phenomena most frequently encountered are muscle spasms chiefly affecting the head and neck, appearing as involuntary spasm of tongue and mouth muscles, spasm of the masseter preventing mouth opening, facial muscle spasm leading to grimacing, and spasm of the orbicularis oculi resulting in excess blinking or blepharospasm. Ocular involvement, oculogyric crisis, may lead to painful upward gaze lasting minutes or hours. Neck involvement, frequently associated with ocular manifestations, consists of torticollis, antecollis or retrocollis. Involvement of trunk (opisthotonos) or limbs, though less frequent, may lead to bizarre gaits or difficulties in walking.

Acute dystonic effects are usually seen in the first 72 hours of neuroleptic treatment. They affect up to $5 \%$ of all patients started on these compounds. 4 The pathophysiology of acute dystonic reactions is unknown, though the clinical manifestations suggest an excess dopaminergic activity, possibly secondary to acute antagonism of dopamine autoreceptors. Acute dystonic reactions are most prevalent in young male patients. "They can occur with any type of neuroleptic but are seen most often in association with the piperazine side chain phenothiazines and the butyrophenones. They are reversible either spontaneously or with treatment. An episode can usually be terminated abruptly by injection of an antiparkinsonian drug. Treatment with neuroleptics can, however, be continued with only a slight risk of recurrence.

\section{AKATHISIA}

This refers to a condition characterized by a subjective sense, frequently accompanied by objective evidence, of restlessness. Although reported in postencephalitic and idiopathic parkinsonism, it is now most frequently encountered in patients on neuroleptic drugs. The condition usually presents in the first month of neuroleptic treatment. A delayed-onset variant, tardive akathisia, is reported in up to $25 \%$ of patients with T1).

Patients with akathisia complain of an inability to sit still. Accompanying features include fidgeting, rocking back and forth, crossing and uncrossing the legs, or constant pacing. Motor activity relieves the inner subjective urge to move and, not surprisingly, the condition is frequently mistaken for agitation. The association of an inner urge and an outward motor response bears some resemblance to a tic, though akathisia differs in that the urge to move is constant and the motor component is continuous, stereotyped, and repetitive. thisia is not understood, but may be relatee blockade of nonstriatal dopaminergic pathw Anticholinergic drugs are often ineffective in lieving the condition. Benzodiazepines may Often, it is necessary to reduce the dosage neuroleptic or substitute another class of pound.

\section{PARKINSONISM}

This is the most common neurologic side fect of neuroleptics, occurring in from 20 to 4 of patients treated with such agents. ${ }^{4,7}$ Symptot which usually appear in the first 3 weeks of ti ment, are both dose-related and drug-related. piperazine phenothiazines and butyrophen are the compounds most often implicated in cipitating this syndrome, whereas the substit benzamides such as sulpiride and thioxanth are least often responsible. The condition is with increasing frequency with age and in t with underlying cerebral disease.

The clinical manifestations are identical those of Parkinson's disease, with tremor, rigi postural abnormalities, and bradykinesia. Droo is a frequent accompaniment. Bradykinesia, earliest manifestation, is usually followed by $\mathrm{E}$ ity, abnormalities of posture, and, lastly, rest tremor. Rigidity and tremor are usually symme cal. An unusual variant seen following medium long-term neuroleptic treatment is the occurre of a 5 to $7 \mathrm{~Hz}$ perioral tremor, so-called rabbits drome. ${ }^{8}$

The mechanism of drug-induced parkin ism is blockade of striatal postsynaptic dopam (D2) receptors, producing a functional deficien of dopamine at those sites. Treatment of the $c$ dition involves either stopping or reducing neuroleptic or alternatively introducing an a cholinergic agent. However, tolerance to the ex pyramidal effects of neuroleptics develops des continuation of an offending agent. This phen enon of tolerance may be related to pharma netic factors, such as increased metabolism. W drawal of the antipsychotic drug usually lead resolution of parkinsonism within a matter weeks, though the condition may persist fot months or longer. ${ }^{10}$ One reason for this appears be the persistent presence of active metabolites? up to 3 months after discontinuation of trea ment."

\section{TARDIVE DYSKINESIA}

TD is a hyperkinetic involuntary movene disorder of diverse and widespread manifestati The occurrence of the syndrome in associabi 
with neuroleptic medication was first described in 1957, shortly after the introduction into clinical practice of phenothiazine neuroleptics. ${ }^{12}$ Further observations of a hyperkinetic syndrome occurring in close temporal association to commencement of neuroleptic medication followed. ${ }^{13-15}$ The term "tardive dyskinesia" to describe this syndrome was introduced by Faurbye. ${ }^{16}$ TD constitutes the most serious side effect of antipsychotic treatment by virtue of its disfiguring nature and its persisience.

The abnormal movements that comprise TD are coordinated, stereotyped, and rhythmic. Most common is cranial involvement, but limb and truncal involvement are also seen. Functional effects of TD include difficulties in speaking, swallowing, and breathing, and abnormalities of posture or gait. The clinical manifestations may be exacerbated by emotional arousal; they disappear during sleep. The earliest cranial manifestation is frequently a writhing movement of the tongue on the floor of the mouth. More marked facial involvement leads to tongue protrusion, often rapid protrusion and withdrawal (so-called flycatcher tongue), grimacing, lateral chewing jaw movements, and sucking or pursing of the lips. These symptoms may appear singly or in combination (buccolinguomasticatory [BL.M] dyskinesia). Limb involvement consists of choreiform or jerky movements that may be accompanied by distal athetoid movements of the limbs. Truncal involvement indudes writhing or ballistic movements, rhythmical movements of the body from side to side or rocking when the subject is seated. Multivariate statisical analyses of these movements identify two subsyndromes of TD, a cephalic and a trunk/limb dyskinesia. ${ }^{17}$ Support for such fractionation comes from observations that the cephalic syndrome responds to increased neuroleptic dosage and worsens with anticholinergic treatment while the peripheral types may improve with anticholinergic and worsen with increased neuroleptic.

Although TD is an undoubted effect of longterm neuroleptic treatment, there is considerable controversy as to whether the occurrence of this rondition in chronic psychiatric patients is due solely to the effects of neuroleptics, to the underlyng disease process, or to an interaction of drugs with the underlying disease state. ${ }^{18}$ Central issues in this controversy are whether a condition akin to ID occurred in the preneuroleptic era and whether the prevalence of the condition has increased with increasing use of neuroleptics. There svery little information on the latter point, though there is considerable clinical evidence that condions characterized by excess movements, akin to ID, were seen in the preneuroleptic era. Kraepelin described abnormal movements in chronic psychotic patients thus: "Connected with these are, further, smacking and clicking of the tongue.... But besides we observe especially in the lip muscles, fine lightning-like or rhythmical twitchings, which in no way bear the stamp of voluntary movements. The same is the case in the tremor of the muscles of the mouth which appears sometimes in speaking...." However, it is uncertain whether these early descriptions are indeed from patients with primary schizophrenic illness or schizophrenic illness secondary to primary neurologic conditions, such as postencephalitic states. Furthermore, TD is described in patients receiving neuroleptics for conditions other than schizophrenia, such as manic-depressive illness. ${ }^{19}$

The reported prevalence of TD in populations of patients receiving neuroleptic medication varies from 1 to $40 \%$. Discrepancies in reported prevalence are partly attributable to variations in operational definitions of TD, with some early reports being based solely on the BLM triad. Other variables that may influence prevalence figures include age, duration, type, and dosage of medication. An accepted prevalence figure is $20 \%$ of patients on long-term neuroleptic treatment. ${ }^{6.20}$

Risk factors for the development of the disorder that have emerged from epidemiologic surveys include age, female sex, and underlying cerebral disease or damage. ${ }^{20.21}$ An increased risk of developing the disorder is also reported in patients receiving concurrent anticholinergic drugs. ${ }^{22,23}$ The disorder is most often seen in schizophrenic patients but also occurs in other populations of neuroleptic-treated patients. Indeed, contrary to arguments for special association with schizophrenia, it has been suggested that patients with affective disorder exposed to neuroleptics are at greater risk of developing the disorder. ${ }^{24}$ The condition is also encountered in postencephalitic states, as a spontaneous condition in up to a quarter of the elderly population, ${ }^{25}$ during L-dopa therapy, and as minor manifestations in Wilson's disease, Huntington's disease, and Sydenham's chorea. A statedependent variant has been reported in association with the depressive phase of bipolar affective disorder. ${ }^{26}$

TD is generally associated with medium to long-term exposure to neuroleptics but can occur with exposure as brief as several weeks. The piperazine side-chain and butyrophenone drugs are associated with an increased risk of developing the disorders while the substituted dibenzamides and newer antipsychotic agents such as clozapine are relatively risk free. The condition may first appear during withdrawal of neuroleptics: withdrawal dyskinesia. There are no consistent findings relat- 
ing dosage of neuroleptic to risk of developing the syndrome.

The disorder needs to be distinguished from stereotypies and mannerisms of schizophrenia. The former are repetitive, pointlessly executed, normal movements, while the latter are bizarre, unnatural exaggerations of normal movements.

TD in its full expression may be persistent. However, reversal of the syndrome occurs in a third of patients on withdrawal of the neuroleptic. ${ }^{27}$ Evidence suggests that such spontaneous remission of TD is inversely correlated with age. ${ }^{28}$ Specific treatments for TD are lacking. An increase in neuroleptic dosage results in temporary suppression of symptoms. In view of a lack of effective treatments the focus must be on prevention. There is little hard evidence that so-called drug holidays - that is, periods off neuroleptics-lead to a decreased risk of developing the condition. In susceptible individuals it is best to use neuroleptics with a decreased risk of inducing the condition, such as sulpiride or clozapine. In patients who have developed the condition, but in whom there are compelling clinical reasons to continue treatment, a switch to one of the low-risk agents is indicated.

The pathophysiology of the condition is not fully understood. Overstimulation of striatal dopamine systems is the mechanism mainly implicated. The theoretical explanation for dopamine overstimulation in the presence of DA antagonists invokes postsynaptic supersensitivity secondary to blockade of DA receptors. ${ }^{29.30}$ Experimental findings from animal models of TD uphold this hypothesis $^{31}$ although other hypotheses have involved alteration of GABA.

\section{TARDIVE DYSTONIA}

Dystonic movements of late onset as a major side effect of neuroleptic medication have been recognized for almost 20 years. ${ }^{32}$ Tardive dystonia is considered a variant of TD rather than a separate clinical entity. Its clinical manifestations are those of a dystonia with sustained involuntary muscular contractions. ${ }^{33}$ Dystonic movements in the form of hyperextension or abduction of limbs, excessive lordosis, and pelvic rotation are frequent accompaniments of TD. In tardive dystonia the dystonic movements are the predominant feature. The condition resembles a primary dystonia and may be focal, segmental or generalized in distribution. A variant is so-called Meige's syndrome, which involves both a lower facial dystonia and blepharospasm, sometimes referred to as the orofacial-mandibular blepharospasm syndrome. sive to drugs. The major risk factor is similar i that of TD, namely, prolonged exposure to ne: roleptics. However, unlike the latter, it is more ff quently encountered in young patients. A relati ship between age of onset and bodily distribut of dystonic movements has been noted, with $\mathrm{g}$ eralized dystonias tending to occur in the yo while segmental or focal dystonias occur in individuals. ${ }^{33}$

The onset of the condition has been describ after intervals of neuroleptic treatment van from 3 days to 11 years. Prevalence data are spar One study of hospitalized psychiatric patients w chronic illness found a figure of approxima $2 \%{ }^{34}$ As in TD, it may be masked by increases remit following decreases or withdrawal of neir leptics. In a proportion of cases it is persistent af withdrawal of drugs. Treatment is unsatisfacto Strategies that may be helpful include antido minergic drugs, anticholinergics, tetrabenazin and benzodiazepines. ${ }^{35}$

\section{NEUROLEPTIC MALIGNANT SYNDROME}

This is an uncommon but potentially fatal effect of neuroleptic medication. Its infreque and variable clinical features often lead to fail in detection. The full-blown syndrome devel over a matter of 48 to 72 hours. In the majority cases onset follows primary exposure to neurole tics, though patients developing neuroleptic ma nant syndrome (NMS) may in some instancesh had previous exposure to neuroleptics. The drome was named in 1968 by Delay ${ }^{36}$ though f reactions in association with neuroleptics were served shortly after their introduction. ${ }^{37.38}$

The clinical features include hypertherm extrapyramidial symptoms such as parkinsonis rigidity, dystonia, and tremor, autonomic insto ity with tachycardia, diaphoresis, lability of blo pressure, tachypneic hypoventilation, alteration consciousness, stupor, or coma. Dehydration is feature of most cases on presentation.

Although most authors accept a pathogen role for neuroleptics, there is a remarkably do resemblance between NMS and fulminating chotic illnesses recognized before the neurolep era. A syndrome of acute catatonic delirium reported as far back as 1832 by Calmeil and sequent descriptions outlined potentially fatal ps chotic illnesses with features that incude hype thermia, catatonia, and alteration of consciousne frequently ending in coma or death. ${ }^{39,40}$ Varyi epithets, including lethal catatonia, catatonic d lirious state, délire aigu, exhaustion syndrom Stauder's lethal catatonia, and hypertoxic schin phrenia have been used to describe this syndrom 
Follow-up studies of these catatonic-delirious states demonstrate an outcome similar to that of NMS. ${ }^{41}$ These early observations raise a question as to whether neuroleptics in themselves are necessary or sufficient causes for the development of NMS. A role for neuroleptics is suggested by the occurrence of the syndrome close to the time that they are started or increased in dosage. This association may, however, be spurious in that most psychotic disorders, which might include conditions previously classified as delirious catatonia, are likely nowadays to be treated early with neuroleptics. More compelling evidence for a role for neuroleptics is that NMS is also seen in nonschizophrenic disorders following exposure to neuroleptics. ${ }^{42.43}$ Furthermore, withdrawal of neuroleptics may lead to recovery while reintroduction may lead to relapse of NMS. ${ }^{44}$ There have been suggestions that NMS can be considered a neuroleptic-induced iatrogenic form of lethal catatonia. ${ }^{45}$

Laboratory findings, which may be entirely normal, include a polymorphonuclear leukocytosis in $70 \%$ of cases, raised muscle creatinine (CK) in $92 \%$, and myoglobinemia in $70 \%$. Abnormalities of liver function tests are also reported in up to $70 \%$, as is proteinuria in $90 \%$ of cases. Raised CK levels are also reported in psychotic patients; this could be construed as evidence that NMS is one facet of spectrum of disorders for which elevated levels of this enzyme are a marker. Evidence of muscle necrosis on biopsy has been reported in up to half of patients affected: Complications of the syndrome include infection, rhabdomyolysis, renal failure (due to rhabdomyolysis and myoglobinemia), and pulmonary insufficiency. Investigations including electroencephalography, computed tomography, and analysis of cerebrospinal fluid are unremarkable. ${ }^{46}$

The prevalence of NMS is difficult to assess given that the condition is often unrecognized. It is most frequently cncountered in association with butyrophenones and piperazine phenothiazines. Estimates of its prevalence among neuroleptictreated populations vary from 0.5 to $1.5 \%{ }^{47}$ The condition is reported as up to three times more common in males. Little is known concerning the primary pathologic features, though striatal and hypothalamic damage have been postulated. ${ }^{48} \mathrm{~A}$ role for dopamine is likely in that the condition is seen with agents other than neuroleptics that alter dopamine function. ${ }^{49}$ The overall mortality from the condition is of the order of 20 to $30 \%$. This may well be falling with earlier recognition and better management. ${ }^{50}$ Withdrawal of neuroleptic leads to recovery generally within 7 to 10 days. Symptomatic measures consist of rehydration, control of temperature, and maintenance of cardio- vascular and renal function. Dopamine agonists are used on the assumption that the syndrome is at least partly due to dopamine blockade. Treatments of this nature with reported beneficial effects include bromocriptine, amantadine, and dantrolene. ${ }^{51,52}$

\section{LITHIUM}

The naturally occurring salt, lithium carbonate, is one of the most effective treatments in psychiatry. Its prime role is in the treatment of cyclic mood disorders, both unipolar and bipolar. Its therapeutic effects are seen with dosages that give a serum level of between 0.5 and $1.2 \mathrm{mEq} /$ liter. Neurologic side effects from lithium are prominent with increasing serum levels above $1.2 \mathrm{mEq} /$ liter. Beyond this range, there develops a coarse tremor, ataxia, dysarthria, drowsiness, hyperreflexia, seizures, myoclonus, coma, and eventually death. ${ }^{53}$ Occasionally, the signs may be unilateral, suggesting an alternative diagnosis. These side effect are well recognized and careful monitoring of serum lithium levels should prevent their development. Less widely recognized are neurologic reactions occurring within the therapeutic range. The most prevalent of these is a fine hand tremor which may be a transient effect on commencement of lithium or a long-term effect. ${ }^{54}$ Less common are instances of parkinsonism. ${ }^{55}$

The most serious neurologic side effects are rare cases of a neurotoxic reaction in patients on combined lithium and neuroleptic therapy, particularly haloperidol. This is characterized by extrapyramidal and cerebellar signs which may be permanent. ${ }^{56-58}$ Lithium used concurrently with neuroleptics in bipolar affective patients may also increase the risk of developing TD in this group. ${ }^{19}$

\section{ANTIDEPRESSANTS}

\section{MONOAMINE REUPTAKE INHIBITORS}

This broad class of agents constitutes the mainstay treatment of affective disorders and includes the tricyclics, tetracyclics, and the newer selective monoamine reuptake inhibitors (MARI), such as 5-hydroxytryptophan (5-HT) reuptake inhibitors. Since this grouping contains a wide diversity of pharmacologic compounds, many with a broad spectrum of central effects, the range of side effects is great. However, as a general rule, these compounds are devoid of any permanent neurologic sequelae. This is somewhat surprising given 
the structural similarity between the tricyclics and the phenothiazine neuroleptics.

Neurologic side effects at toxic levels or overdosage are largely attributable to anticholinergic effects, which include confusion, distractability, hallucinations, slurred speech, insomnia, and coma. ${ }^{59}$ At therapeutic levels, like the neuroleptics, the tricyclics and tetracyclics are associated with peripheral antimuscarinic effects, including dry mouth, blurred vision, delayed ejaculation, and urinary retention. Trazodone may cause priapism and permanent impotence. ${ }^{60}$ Central effects are mainly dose related and are seen especially in the elderly. Confusion, reported in $10 \%$ of patients, is possibly related to the anticholinergic effects of some MARIs. ${ }^{61}$ A fine tremor is seen in approximately $5 \%$ of patients on tricyclics, but extrapyramidal effects proper are extremely rare. Alteration of seizure threshold is a potential side effect of all drugs in this class. Isolated cases of NMS are also reported in association with this class of antidepressants.

\section{MONOAMINE OXIDASE INHIBITORS}

This chemically broad category of drugs shares a common property of inhibiting oxidative deamination of monoamines. Two subclasses are recognized, with relatively selective potencies for inhibition of monoamine oxidase (MAO) A and $\mathrm{B}$, the two subtypes of MAO enzyme. All compounds in this group are irreversible ("suicide") inhibitors of MAO.

Toxic effects are seen following overdosage and at therapeutic levels. The former consist of agitation, hyperreflexia, hyperpyrexia, hallucinations, and convulsions. At therapeutic levels, neurologic side effects include tremor, insomnia, hallucinations, and confusion. Peripheral neuropathy is seen in a proportion of patients exposed to the hydrazine group of MAO inhibitors.

\section{ANTIANXIETY AGENTS}

The main compounds in clinical use for the management of anxiety states are the benzodiazepines. Indeed, they comprise the most widely prescribed psychotropic agents. Newer antianxiety compounds, particularly with affinities for central serotonergic receptors, are under evaluation in clinical practice. The principal adverse effects of these agents relate to their potential for causing dependence and abuse. Therefore a physical withdrawal syndrome, of which seizures may be a component, is frequently encountered in patients taking these compounds on a medium to long- term basis. In patients stabilized on these con pounds there are remarkably few side effec Neurologic side effects are mild and consist of paired psychomotor performance and impairm of attention on vigilance tasks. ${ }^{62.63}$

\section{ELECTROCONVULSIVE THERAPY}

Since its introduction into psychiatry treatment has generated a great deal of versy with regard to both its efficacy and its effects. Neurologic side effects are few. They sist principally of memory disturbance. Co brain damage as a result of ECT has freque been claimed but remains unproven.

Transient memory loss, both retrograde an anterograde, is a well recognized and uncon verted immediate side effect of ECT. A major con ponent of these deficits can be attributed to the fect of severe depression per se on men functioning. Long-term memory impairment frequent subjective complaint of patients whol received ECT. An objective basis to these plaints is established. A disturbance of per biographic memory is seen in a proportion of tients following ECT. ${ }^{64.65}$ It is less marked with lateral, nondominant hemisphere (as opposed bilateral) ECT.

\section{PSYCHOSURGERY}

Psychosurgery is now relatively rare in chiatry. In clinical practice, however, one still e counters patients who have been exposed to type of intervention. In Great Britain the numb of procedures carried out fell from 70 in 1979 15 in 1986. It remains a highly controversial inte vention in view of the nature of the procedure, scientific justification, and the ethical issues volved. Many practicing psychiatrists disagree tirely with its use. Those who see a role for psych surgery limit its usage to patients with severe intractable anxiety, obsessional disorders, and pressive illness that has not responded to therapies. Procedures now in use are entirely reotactic, producing lesions of the inferior from white matter (subcaudate tractotomy) and ante cingulate gyri. The former procedure is formed for affective disorders, anxiety, or dep sion, while the latter is indicated in intractable sessional disorders.

There are many problems in evaluating ne rologic side effects of psychosurgery particularl one is dealing with an unusual and atypical po lation. Furthermore, there is a striking paucit 
Independent studies evaluating the effectiveness and outcome of the procedure. Most data are therefore from centers with a vested interest in the procedure. Epilepsy is reported in up to $3 \%$ of paients who have had the procedure. Personality thange has been reported in up to $7 \% .{ }^{66}$ Curiously, lor a procedure that damages cerebral tissue, there ireno reports of a fall in intelligence or more focal hatellectual deficits.

\section{CONCLUSIONS}

The overall frequency of neurologic side efts resulting from psychiatric treatments is low then viewed in the context of the widespread use of psychotropic agents in developed countries. among psychotropic drugs, the most hazardous, with regard to neurologic side effects, are the neumoleptics. Although side effects from neuroleptics are generally reversible, there are a number of responses that may be irreversible or even fatal. This makes imperative the judicious use and careful supervision of such compounds. However, the potenial benefits of neuroleptics in the treatment of the psychoses still far outweigh any risks associated with their use.

The development of more specific treatments or the psychoses is a major endeavour within psyhiatry and the pharmaceutical industry. Advances n neuroimaging and molecular genetics are havng a major impact on the scientific underpinnings f psychiatry, leading to the acquisition of more recise knowledge concerning pathophysiologic mechanisms. The impact of such knowledge on herapeutic developments is difficult to gauge at present. Therapeutic advances in psychiatry, until now, have largely been due to serendipity, though there is every reason to feel optimistic that treatments based on precise knowledge of disease processes are within our grasp.

\section{REFERENCES}

Delay J, Deniker P, Harl JM. Utilisation en therapeutique psychiatrique d'une phenothiazine d'action centrale elective. Ann Mcd Psychol (Paris) 1952;110:112-7

Labhardt F. Technik, Nebenerscheinungen und Komplikationen der Largactiltherapic. Schweiz Arch Neurol Psychiatr 1954;73:338-45

Delay J, Deniker P, Ropert H, et al. Syndromes neurologiques experimentaux et therapeutique psychiatrique. Presse Med 1959;67:123-6, 201-3

Marsden CD, Tarsy D, Baldessarini RJ. Spontaneous and drug induced disorders in psychotic patients. In: Belson DF, Blumer D, eds. Psychiatric aspects of neurologic diseasc. New York: Grunc \& Stratton 1975: 219-66
5. Ayd F. A survey of drug-induced extrapyramidal reactions. JAMA 1961;175:1054-60

6. Marsden CD, Fahn S. Problems in the dyskinesias. In Marsden CD, Fahn S, eds. Movement disorders 2. Butterworth 1987

7. Crane GE. Persistent dyskinesia. Br J Psychiatry 1973; 122:395-405

8. Jus K, Jus A, Gautier J, et al. Studies of the actions of certain pharmacological agents on tardive dyskinesia and on the rabbit syndrome. Int J Clin Pharmacol 1974;9:138-45

9. Loga S, Curry S, Lader M. Interactions of orphenadrine and phenobarbitone with chlorpromazine: plasma concentration and effects in man. Br J Clin Pharmacol $1975 ; 2: 197-208$

10. Gardos G, Cole J, Tarsy D. Withdrawal syndromes associated with antipsychotic drugs. Am J Psychiatry 1978; 135:1321

11. Forrest I, Forrest F. Urine color test for the detection of phenothiazine compounds. Clin Chem 1960;6:11-15

12. Schonecker M. Ein eigentumliches Syndrom im oralen Bereich bei Megaphen-applikation. Nervenarzt 1957; 28:35-42

13. Sigwald J, Bouttierd, Raymondeaud C, Piot C. Quatre cas de dyskinesie facio-linguo-masticatrice à evolution prolongée secondaire à un traitément neuroleptique. Rev Neurol (Paris) 1959;100:751-5

14. Hunter R, Earl CJ, Thornicroft S. An apparently irreversible syndrome of abnormal movements following phenothiazine medication. Proc R Soc Med 1964;57: $758-62$

15. Degkvitz R, Luxenburger O. Das terminale extrapyramidale Insuffizienz bzw. Defektsyndrom infolge chronischer Anwendung von Neuroleptika. Nervenarzt 1965;36:173-5

16. Faurbye A, Rasch PJ, Petersen PB, et al. Neurological symptoms in pharmacotherapy of psychosis. Acta Psychiatr Scand 1964;40:10-27

17. Kidger T, Barnes T, Trauer T, Taylor EJ. Sub-syndromes of tardive dyskinesia. Psychol Med 1980;10:513-20

18. Crow TJ, Owens DGC, Johnston EC, et al. Does tardive dyskinesia exist? Mod Probl Pharmacopsychiatry 1983; $21: 206-19$

19. Dinan TJ, Cohen D. Tardive dyskinesia in bi-polar affective disorder: relationship to lithium therapy. $\mathrm{Br} J \mathrm{Psy}$ chiatry $1989 ; 155: 55-7$

20. Kane J, Smith JM. Tardive dyskinesia: prevalence and risk factors 1959-1979. Arch Gen Psychiatry 1982;39: $473-81$

21. Smith JM, Oswald WT, Kucharski T, et al. Tardive dyskinesia: age/sex differences in hospitalised schizophrenics. Psychopharmacology 1978;58:207-11

22. Fann WE, lake CR, Gerber CJ, et al. Cholinergic suppression of tardive dyskinesia. Psychopharmacology 1974;37:101-7

23. Gerlach J. Reisby N, Randrup A. Dopaminergic hypersensitivity and cholinergic hypofunction in the pathophysiology of tardive dyskinesia. Psychopharmacology $1974 ; 34: 21-35$

24. Kane J, Struve FA, Weinhold P. Strategy for the study of patients at high risk for tardive dyskinesia. Am J Psychiatry $1980 ; 137: 1265-7$

25. Blowers AJ. Epidemiology of tardive dyskinesia in the elderly. Neuropharmacology 1981;20:1339-40

26. Cutler NR, Post RM, Rey AC, Bunney WE. Depressiondependent dyskinesias in two cases of manic-depressive illness. N Engl J Med 1983;18:1088-9

27. Jeste DV, Wyatt RJ. Therapeutic strategies against tardive dyskinesia. Arch Gen Psychiatry 1982;39:803-16

28. Smith JM, Baldessarini RJ. Changes in prevalence, severity, and recovery in tardive dyskinesia with age. Arch Gen Psychiatry 1980;37:1368-73

29. Carlsson A. Biochemical implications of dopa-induced actions on the central nervous system with particular reference to abnormal movements. In: Barbeau A, 
McDowell FH, eds. L-Dopa and parkinsonism. Philadelphia: FA Davis, 1970;205-3

30. Klawans HL. The pharmacology of tardive dyskinesias. Am J Psychiatry 1973;130:82-6

31. Clow A, Jenner P, Marsden CD. An experimental model of tardive dyskinesia. Life Sci 1978:23:421-4

32. Keegan DL, Rajput AH. Drug induced dystonia tarda: treatment with L-dopa. Dis Nerv Sys 1973;38:167-9

33. Burke RE, Fahn S, Jankovic J, et al. Tardive dystonia: late-onset and persistent dystonia caused by antipsychotic drugs. Neurology (NY) 1982;32:1335-46

34. Kang UJ, Burk RE, Fahn S. Natural history and treatment of tardive dystonia. Mov Disord 1986;36(Suppl 1): 121

35. Freedman JH, Kucharski TL, Wagner RL. Tardive dystonia in a psychiatric hospital. J Neurol Neurosurg P'sychiatry 1987;50:801-3

36. Delay J, Deniker P. Drug induced extra-pyramidal syndrome. In: Vinken N, Bruyn G, eds. Handbook of clinical neurology. New York: Flsevicr 1968;6:248-66

37. Ayd FJ, Junior JR. Fatal hyperpyrexia during chlorpromazine therapy. J Clin Psychiatry 1956;27: 189-92

38. Preston J. Central nervous system reaction to small doses of tranquilizers. Am Pract Dig Treat 1959; 10:627-30

39. Aronson MJ, Thompson SB. Complications of acute catatonic excitement. Am J Psychiatry 1950;107:216-20

40. Kellam AM. The neuroleptic malignant syndrome, so called: survey of the world literature. Br I Psychiatry 1987;150:752-9

41. Laskowska D, Urbanik K, Jus A. The relationship between catatonic-delirious state and schizophrenia, in the light of a follow-up study (Stauder's lethal catatonia). Br J Psychiatry 1965;1 1 1:254-7

42. Smith JA, Carter JH. Neuroleptic malignant syndrome with a positive Weil-Felix test. Am J Psychiatry 1984; 141:609

43. Konikoff F, Kuritzky A, Jerushalmi Y, Theodor E. Neuroleptic malignant syndrome induced by a single injection of haloperidol. Br Med J 1984;289:1228-9

44. Bourgeois M, Tignol J, Henry P. Syndromes malins et morts subites au cours des traitements par neuroleptiques simples et retard. Ann Med Psychol (Paris) 1971; 2:729-46

45. Mann S, Caroff SN, Bleier HR, et al. Lethal catatonia. Am J Psychiatry 1986;143:1374-81

46. Caroff S. The neuroleptic malignant syndrome. J Clin Psychiatry 1980;41:79-83

47. Cohen BM, Baldessarini RJ, Pope HG, Lipinski JF. Neuroleptic malignant syndrome. N Fngl J Med 1985; 313:1293

48. Henderson VW, Wooten GF. Neuroleptic malignant syn- drome. A pathogenetic role for dopamine recep blockade? Neurology (NY) 1981;31:132-7

49. Burke RE, Fahn S, Mayeux R, et al. Neuroleptic nant syndrome caused by dopamine depleting dru a patient with Huntington's chorea. Neurologt 1981:31:1022-6

50. Mucller P, Vester J, Fermaglich J. Neuroleptic malig syndrome. JAMA 1983;249:386-8

51. Gramato JE, Stern BJ, Ringel A, et al. Neurolepticn nant syndrome: successful treatment with dantroi bromocriptine. Ann Neurol 1983;14:89-90

52. Delacour JL, Daoudal P, Chapoutot JL, Roc QB. ment du syndrome malin des neuroleptiques dantrolene. Nouv Presse Med 1981;10:3572-3

53. Saron BM, Gaind R. Lithium. Clin Toxicol 1973;6; 69

54. Johns S, Harris B. Tremor. Br Med J 1984;288:19

55. Tyrer P, et al. Extrapyramidal symptoms in two pat receiving lithium carbonate which worsened folle administration of orphenadrine. Br J Psychiatry 136-91

56. Cohen WJ, Cohen NH. Lithium carbonate, halope and irreversible brain damage. JAMA 1974: 1283-7

57. Thornton WE. Thornton BJP. Lithium intoxicarin report of two cases. Can Psychiatr Assoc J 1975 $281-2$

58. Loudon JB, Waring $\mathrm{H}$. Toxic reactions to lithim: haloperidol. Lancet 1976;2:1088

59. Shopsin B, Waters B. The pharmacotherapy of ma pressive syndrome. Part 1: Treatment of acuted sion. Psychosomatics 1980;21:542

60. Lansky MR, Selzer J. Priapism associated with trazodi therapy: case report. Clin Psychiatry 1984;45:232-

61. Davies RK, Tucker GJ, Horrow M, Detre TP. Confusi episodes and anti-depressant medication. Am J chiatry 1971;128:95-99

62. Betts T, Birtelc J. Effects of two hypnotic drugs on driving performance next morning. $\mathrm{Br}$ Med $\mathrm{J}$ 285:852

63. Holstrom C, Lader MH. Benzodiazepine withdra phenomena. Int Pharmacopsychiatry 1981;16:235-

64. Squire LR, Slater PC, Miller PI.. Retrograde amnesiz bilateral electroconvulsive therapy. Long-term for up. Arch Gen Psychiatry 1981:38:89-95

65. Weiner RD, Rodger HJ, Davidson JRT, Squire LR fects of stimulus parameters on cognitive side eff Ann NY Acad Sci 1986;462:315-25

66. Goktepe EO, Young LB, Bridges PK. A further revit the results of stereotactic subcaudate tractotoms. Psychiatry $1975 ; 126: 270-80$ 